\title{
Tracheal obstruction in a child with a posterior mediastinal mass
}

\author{
Hussein Elattar, MD · James Armstrong, MD · Jerrold Lerman, MD · \\ Michael Pizzuto, MD $\cdot$ Puneet Gupta, MD
}

Received: 22 December 2015/Accepted: 11 January 2016/Published online: 19 January 2016

(C) Canadian Anesthesiologists' Society 2016

Nearly complete tracheal obstruction is a potential catastrophe in a child. It is not commonly associated with a posterior mediastinal mass or a lymphoma. A five-yearold boy presented with chest pain and shortness of breath. Computed tomography (CT) scanning revealed tracheal obstruction above the carina. Rigid bronchoscopy, which was performed under an inhalational anesthetic, confirmed $>90 \%$ tracheal obstruction (Figure, left panel). After an uncuffed tube was carefully positioned beyond the tumor, magnetic resonance imaging (Figure, right panel) delineated a massive posterior mediastinal tumor that extended into the prevertebral space and compressed the posterior tracheal wall just above the carina. It almost completely occluded the lumen. CT-guided paraspinal tissue biopsy confirmed a B-cell Burkitt's lymphoma, for which chemotherapy effectively resolved all symptoms, including the narrowed airway.

This report raises several important issues for pediatric anesthesiologists. First, and contrary to conventional teaching, lymphomas, even the rapidly growing

H. Elattar, MD · J. Armstrong, MD · J. Lerman, MD ( $ه)$ Department of Anesthesiology, Women \& Children's Hospital of Buffalo, SUNY at Buffalo, Buffalo, NY, USA

e-mail: jlerman@rogers.com

MichaelPizzuto, MD

Department of Otolaryngology, Women \& Children's Hospital of Buffalo, SUNY at Buffalo, Buffalo, NY, USA

P. Gupta, MD

Department of Radiology, Women \& Children's Hospital of Buffalo, SUNY at Buffalo, Buffalo, NY, USA 

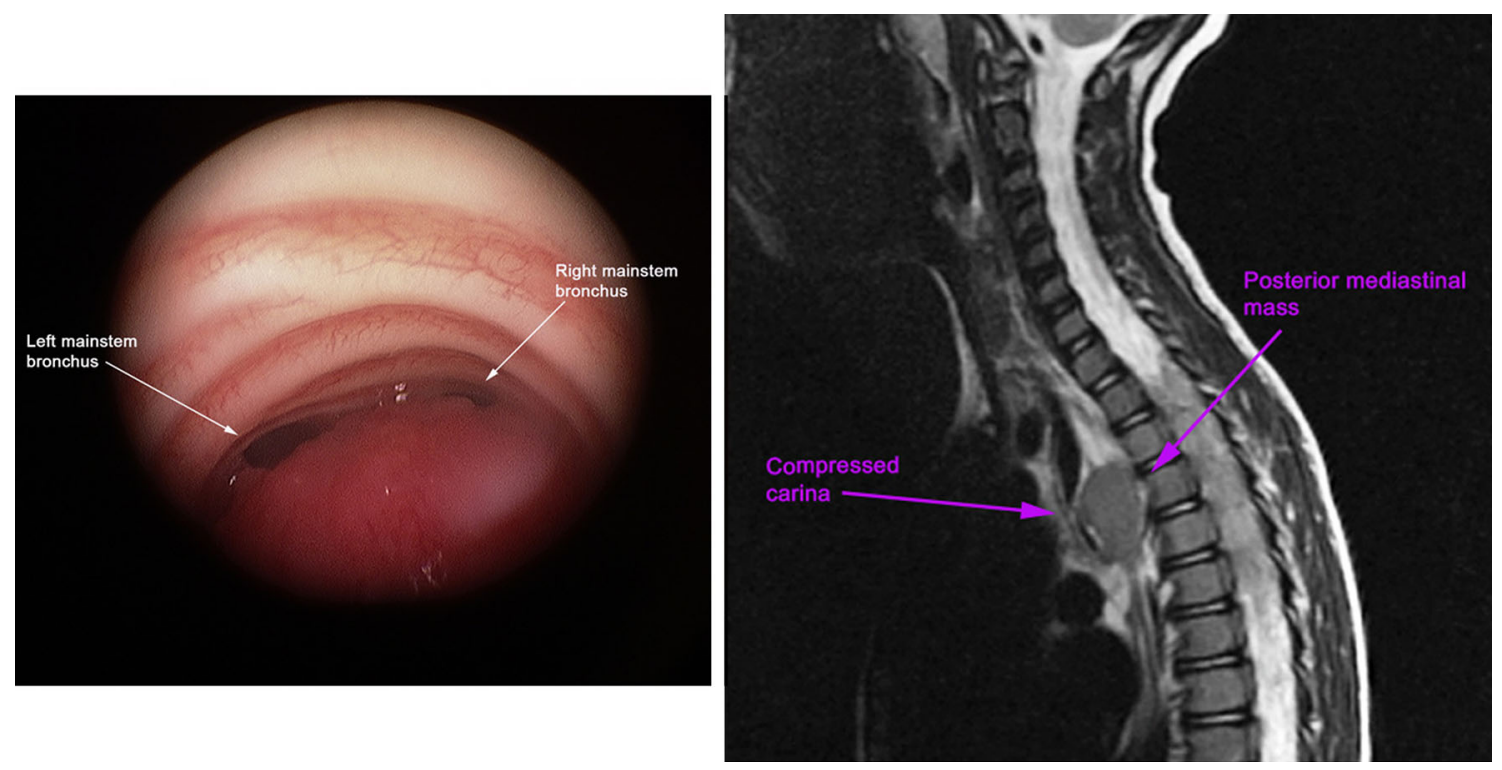

Figure Photograph (left panel) obtained during rigid bronchoscopy shows a posterior mediastinal lymphoma extrinsically compressing the trachea just above the carina. The lymphoma was also investigated using magnetic resonance imaging (right panel)

lymphoblastic T-cell lymphoma, may arise in the posterior mediastinum (and spine). ${ }^{1,2}$ Second, the tracheal lumen may be severely compromised with a posterior mediastinal tumor, a concern not widely recognized. Third, until the tumor type has been confirmed, steroids should be avoided to preclude tumor necrosis, subsequent difficulty with diagnosis confirmation, and an associated tumor lysis syndrome. ${ }^{3}$

\section{Conflicts of interest None declared.}

\section{References}

1. Ranganath SH, Lee EY, Restrepo R, Eisenberg RL. Mediastinal masses in children. AJR Am J Roentgenol 2012; 198: W197-216.

2. Chaari Z, Charfi S, Hentati A, Ayadi I, Abid H, Frikha I. Primary Burkitt lymphoma in the posterior mediastinum. Asian Cardiovasc Thorac Ann 2015; 23: 1110-2.

3. McDonnell C, Barlow R, Campisi P, Grant R, Malkin D. Fatal peri-operative acute tumour lysis syndrome precipitated by dexamethasone. Anaesthesia 2008; 63: 652-5. 